\title{
Medical Imaging with a Microwave Tomographic Scanner
}

\author{
LUIS JOFRE, MEMBER, IEEE, MARK S. HAWLEY, ANTONI BROQUETAS, MEMBER, IEEE, \\ ELÍAS DE LOS REYES, MEMBER, IEEE, MIGUEL FERRANDO, MEMBER, IEEE, \\ AND ANTONIO R. ELIAS-FUSTÉ, MEMBER, IEEE
}

\begin{abstract}
A microwave tomographic scanner for biomedical applications is presented. The scanner consists of a 64 element circular array with a useful diameter of $20 \mathrm{~cm}$. Electronically scanning the transmitting and receiving antennas allows multiview measurements with no mechanical movement. Imaging parameters are appropriate for medical use: a spatial resolution of $7 \mathrm{~mm}$ and a contrast resolution of $1 \%$ for a measurement time of $3 \mathrm{~s}$. Measurements on tissue-simulating phantoms and volunteers, together with numerical simulations, are presented to assess the system for absolute imaging of tissue distribution and for differential imaging of physiological, pathological, and induced changes in tissues.
\end{abstract}

\section{INTRODUCTION}

$\mathrm{F}$ ORMING images of the human body using ionizing radiation has been a part of the diagnostic process in medicine for many years. More recently, other probing radiations, notably ultrasound, have provided successful diagnostic images. Tomography is now in widespread use in the clinic with ionizing radiations (X-ray, isotopes) and with nuclear magnetic resonance imaging. In the past few years, other probing radiations have been considered for tomography, including ultrasound [1], [2], very low-frequency electromagnetic fields in electrical impedance tomography [3], and microwaves [4], [5].

Active microwave imaging consists of illuminating the body to be imaged with a low-power coherent microwave field and measuring the field scattered by the body on the opposite side (transmission imaging) or on the same side (reflection imaging) as the illuminator. The measured data can be processed using specialized reconstruction algorithms to give information on the complex dielectric permittivity of the scattering body. In microwave imaging, the complex electromagnetic field is measured and the reconstruction is also complex, thus much information is available and different parameters can be represented in

Manuscript received August 8, 1988; revised May 8, 1989. This work was supported by CAICYT (Spanish Committee for Scientific and Technical Research Grant 1165-84), FISS (Spanish National Institute of Health Grant 84/2112), Spanish-French Cooperation Program Grant 30/135, and Spanish-British Cooperation Program Grant 17/173. M. Hawley held a fellowship from the Royal Society, U.K.

The authors are with ETSE Telecomunicació, Universitat Politécnica de Catalunya, 08080 Barcelona, Spain.

IEEE Log Number 8933066. image form. Microwave imaging has some potentialities, mainly resulting from the specificity of the interaction mechanisms with the living tissues, different from those encountered in any other technique. Additional advantages include the fact that the probing radiation is not harmful at the low powers employed, and that the technology is that used in the communications field and thus is easily available and relatively inexpensive.

The human body exhibits large variations in the dielectric properties of its various tissue types (Table I). For this reason, microwave diffraction tomography is expected to give information on the distribution of tissue types within the body in image form. This type of imaging is known as absolute imaging. In addition, the dielectric properties of tissue can vary substantially with alterations in the physiological parameters, such as blood flow [6], or with externally induced alterations such as tissue temperature during hyperthermia treatment of cancer [7]. Performing differential imaging is therefore expected to give information on these alterations.

The microwave frequency chosen for imaging of biological objects is a compromise between the losses in tissue and the spatial resolution, which is approximately half a wavelength in the surrounding medium. The useful range is found to be $1-4 \mathrm{GHz}$ depending on the considered application. Among the reasons for the late development of microwave imaging were the a priori ideas about the difficult penetration of microwaves in living tissues, their high attenuation, and their poor resolution. Larsen and Jacobi [8] solved this problem by submerging the body to be imaged in a medium with similar electrical properties, in that case water, improving the spatial resolution by around nine times while simultaneously matching the incident illumination to the body. They produced the first images of biological objects (isolated canine kidneys), the microwave images showing up the internal structure well. Ermert and Dohlus [5] performed computer simulations and measurements on cylindrical objects using mechanically-scanned antennas in order to assess microwave diffraction tomography for imaging of biological objects. Their conclusions were fairly pessimistic due to the breakdown of the approximations used in the reconstruction algorithms for high-contrast objects 
TABLE I

Dielectric Properties of Tissue-Simulating Phantom Materials [21], [24], [25]

\begin{tabular}{|c|c|c|c|c|c|c|}
\hline \multirow[b]{3}{*}{ Tissue } & \multicolumn{4}{|c|}{ Permittivity } & \multirow[b]{3}{*}{ Form } & \multirow{3}{*}{$\begin{array}{l}\text { Composition of } \\
\text { Phantom Material }\end{array}$} \\
\hline & \multicolumn{2}{|c|}{ Tissue } & \multicolumn{2}{|c|}{$\begin{array}{l}\text { Phantom } \\
\text { Material }\end{array}$} & & \\
\hline & $\epsilon^{\prime}$ & $\epsilon^{\prime \prime}$ & $\epsilon^{\prime}$ & $\epsilon^{\prime \prime}$ & & \\
\hline Bone/Fat & $\begin{array}{l}4.50 \\
\text { to } \\
8.35\end{array}$ & $\begin{array}{c}1.17 \\
\text { to } \\
1.60\end{array}$ & 5.1 & 1.1 & Solid & Plaster \\
\hline \multirow[t]{2}{*}{ Muscle } & $\begin{array}{l}49.0 \\
\text { to }\end{array}$ & $\begin{array}{l}16.2 \\
\text { to }\end{array}$ & 54.5 & 17.2 & Liquid & Water(65\%) Ethyl.Alc(35\%) \\
\hline & 58.0 & 19.0 & 53.7 & 19.4 & Gel & $\begin{array}{l}\text { Water( } 65.85 \%) \operatorname{Sugar}(30 \%) \\
\operatorname{NaN}_{3}(0.15 \%) \operatorname{Agar}(4 \%)\end{array}$ \\
\hline $\begin{array}{l}\text { Brain } \\
\text { Gray matter }\end{array}$ & 46.0 & 19.0 & 48.5 & 17.3 & Gel & $\begin{array}{l}\text { Water }(60.85 \%) \text { Sugar( }(35 \%) \\
\operatorname{NaN}_{3}(0.15 \%) \text { Agar( }(4 \%)\end{array}$ \\
\hline White matter & 32.3 & 8.9 & $\begin{array}{l}31.6 \\
31.9 \\
33.4\end{array}$ & $\begin{array}{l}13.5 \\
16.4 \\
8.8\end{array}$ & $\begin{array}{l}\text { Liquid } \\
\text { Liquid } \\
\text { Liquid }\end{array}$ & $\begin{array}{l}\text { Water }(40 \%) \text { n-Prop.Alc }(60 \%) \\
\text { Water }(30 \%) \text { Ethil.Alc(70\%) } \\
\text { Water }(50 \%) \text { Oil }(50 \%)\end{array}$ \\
\hline \multirow[t]{2}{*}{ Blood } & \multirow[t]{2}{*}{58.0} & \multirow[t]{2}{*}{15.6} & 58.0 & 16.1 & Liquid & Water $(80 \%)$ n-Prop.Alc $(20 \%)$ \\
\hline & & & 64.3 & 14.8 & Gel & $\begin{array}{l}\text { Water }(80.85 \%) \text { Sugar }(15 \%) \\
\mathrm{NaN}_{3}(0.15 \%) \text { Agar }(4 \%)\end{array}$ \\
\hline
\end{tabular}

into which category falls the human body. This problem remains the greatest limitation of this technique. An additional drawback of their measurements was the use of a mechanically-scanned measurement system which led to very long measurement times.

Fast techniques for measuring microwave fields have been considered [9], [10]. Bolomey et al. developed a planar system for producing tomograms of submerged bodies and isolated animal organs were again successfully imaged. Subsequent measurements on phantoms and biological objects, in addition to new methods for interpreting data, have shown the possibility of using this technique to produce absolute images of limbs and also differential image useful for monitoring hyperthermia treatments [11], [12].

Research in medical imaging began in Barcelona in 1982 , in close agreement with the approach of Bolomey [13]. In 1985 the work was addressed towards a cylindrical microwave scanner, with a first prototype system being completed in 1986 [14]. Since then studies have been carried out to assess the imaging capability of the system for biomedical applications [15].

\section{The Cylindrical Measurement System}

The prototype measurement system is shown in Fig. 1. A ring of antennas is placed around a tank filled with deionized water into which the object is placed [16]. The water is necessary for matching purposes and to improve the system spatial resolution. The antenna array consists of a circular array of 64 water-loaded waveguide antennas, flared in the $E$ plane forming a conformed sectorial horn $(2.5 \mathrm{~cm}$ height, $2.5 \mathrm{~cm}$ length $)$ providing a collimated field pattern of about $2 \mathrm{~cm}$ in the vertical plane. In the $H$ plane the antenna is an open waveguide with a quasiomnidirectional field pattern. The array is divided into four sectors of 16 antennas. Each subarray of 16 elements has a one-to-16 multiplexer consisting of a tree of p-i-n diodes, allowing one element at a time to be selected. The antennas are connected to the emit/receive equipment through a nonreciprocal two- to four- way switching matrix, allowing selection of each sector as emit or receive.

The emit/receive subsystem provides an emitted signal of one watt at $2.45 \mathrm{GHz}$ at its output, giving a power density of less than $0.1 \mathrm{~mW} / \mathrm{cm}^{2}$ at the body, and receives with a noise figure smaller than $3 \mathrm{~dB}$. A coherent phase quadrature detector allows the measurement of the magnitude and phase of the signal from the selected receive antenna. The system employs the method of modulation and synchronous detection to give a high sensitivity, and minimize unwanted coupling.

The data acquisition routine consists in addressing one of the 64 antennas as emitter and then scanning the scattered field with the elements belonging to the half array opposite the emitter antenna. This procedure is sequentially repeated for each emitter until one revolution is completed. The acquisition is controlled by a 32 bit HP 320 microcomputer which also addresses the A/D converters to digitize the data. The microcomputer performs the reconstruction of the image, Section III, with a processing time of $37 \mathrm{~s}$ and finally displays the result in a high-resolution color screen. The complex value of the image can be displayed in several formats as magnitude/ phase or real/imaginary parts. A linear correspondence relates the image values from minimum to maximum to a colour scale going from blue to red.

Before a measurement is performed, the system is calibrated by comparing the field diffracted by a known object (a centrally-located metal cylinder) with theoretically calculated values. The object is then placed centrally in the water tank, and a measurement performed. The system imaging parameters are given in Table II. The minimum object permittivity change detectable (contrast resolution) and the minimum object temperature change detectable (temperature resolution) refer to experimentally measured values in the water tank, with a measurement time of $3 \mathrm{~s}$. The spatial resolution is given theoretically as a consequence of the algorithm. In practice, the spatial resolution is slightly deteriorated due to a low-pass filter effect resulting from the attenuation of high-order spatial frequencies and is measured to be around $10 \mathrm{~mm}$. For differential imaging, it is often convenient to perform the measurement rapidly. Using an integration time of 1.5 $\mathrm{ms}$ per measurement the acquisition is completed in $3 \mathrm{~s}$. For absolute imaging the measurement time is not very crucial, the limit being the time for which a patient can remain still, say one minute. Component settling times can be extended to improve system stability and measurements can be averaged to reduce noise. For the measurements of phantoms presented in Sections V and VI, a total measurement time of $45 \mathrm{~s}$ was used. 

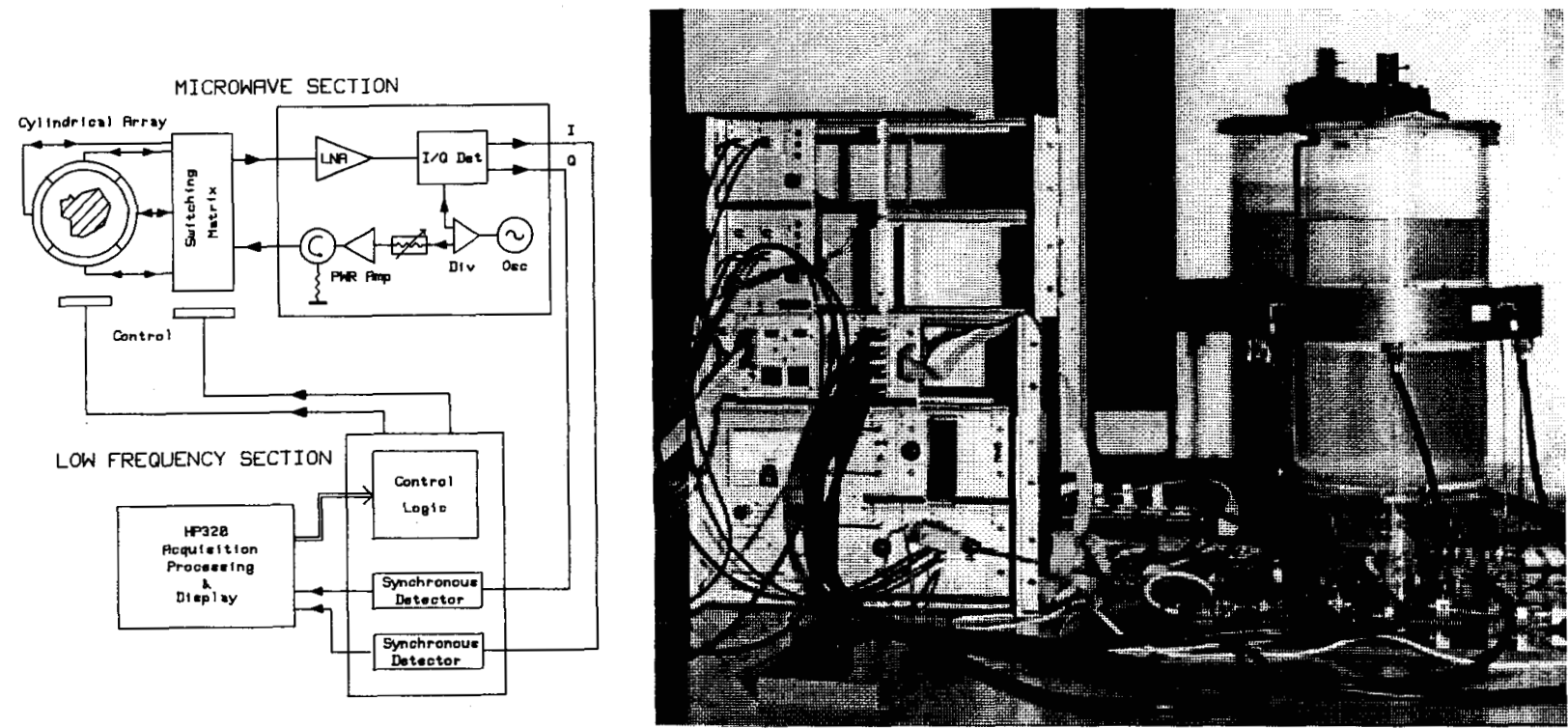

Fig. 1. Prototype cylindrical microwave tomographic scanner.

TABLE II

Prototype Microwave TOMOgRaphic SCANNERSYSTEM PARAMETERS

\begin{tabular}{ll}
\hline SYSTEM PARAMETERS & \\
Frequency & $2.45 \mathrm{GHz}$ \\
Power Density & $<0.1$ \\
& $\mathrm{~mW} / \mathrm{cm}^{2}$ \\
Useful Diameter & $20 \mathrm{~cm}$ \\
Data Acquisition Time & $3 \mathrm{~s}$ \\
Reconstruction Time & $37 \mathrm{~s} \mathrm{on} \mathrm{HP320}$ \\
& \\
IMAGE & $7 \mathrm{~mm}$ \\
Spatial Resolution & $1 \%$ \\
Contrast Resolution & $0.5^{\circ} \mathrm{C}$ \\
Temperature Resolution &
\end{tabular}

\section{Reconstruction Algorithms}

The reconstruction algorithm is formulated in two dimensions and makes use of the Born approximation, which assumes that the scattering acts as a small perturbation on the illumination, and therefore the field within the body is approximated by the incident field. The spectrum of the planewave induced currents in the object is obtained from measurements on a circular line with a set of cylindrical wave illuminations. This is realized with a double convolution operator, efficiently implemented as a double product in the spectral domain [17]. The first convolution synthesizes an incident plane wave as a superposition of cylindrical waves generated by point sources. Using the reciprocity theorem, the planewave scattering amplitude can be obtained from a second convolution of the scattered fields, measured along a cylindrical array. In this way, the problem is reduced to a conventional reconstruction in linear geometries [1], [18], [19] where, assuming weak scattering, the spectral domain of the ob- ject is filled in arcs of radius $K o$ where $K o$ is the wavenumber in the embedding medium. This means that the imaged object is a low-pass version of the original, with a resolution of approximately half a wavelength. Unfortunately, most biological bodies cause the breakdown of the Born approximation due to their high contrast characteristics and large size in terms of wavelengths. For this reason the absolute image obtained is qualitative, i.e., does not reconstruct the original permittivity values, although the image still supplies information on the internal structure of bodies. Differential imaging, Section VI, is carried out by processing the difference between the scattered fields of the reference and perturbed objects. This is equivalent to subtracting images, given the linearity of the algorithm. Differential techniques allow the visualization of small changes in objects which are far beyond the limit of the Born approximation [15], [20].

The reconstruction is obtained in two complex formats, either directly as object permittivity or as object profile, defined as $o=1-\epsilon(r) / \epsilon O$, which shows the contrast between object permittivity $\epsilon(r)$ and the permittivity of the embedding medium $\epsilon$.

\section{Biological Models}

A program of work has been carried out to define appropriate models to simulate the electrical properties of parts of the human body at microwave frequencies. Solid and liquid materials have been produced with dielectric properties simulating most tissue types at $2.45 \mathrm{GHz}$ [21], [24], [25] (Table I). Dielectric properties were confirmed by a reflection method, terminating an open coaxial line with a sample of the material and measuring the complex reflection coefficient very accurately with a HP8510B au- 


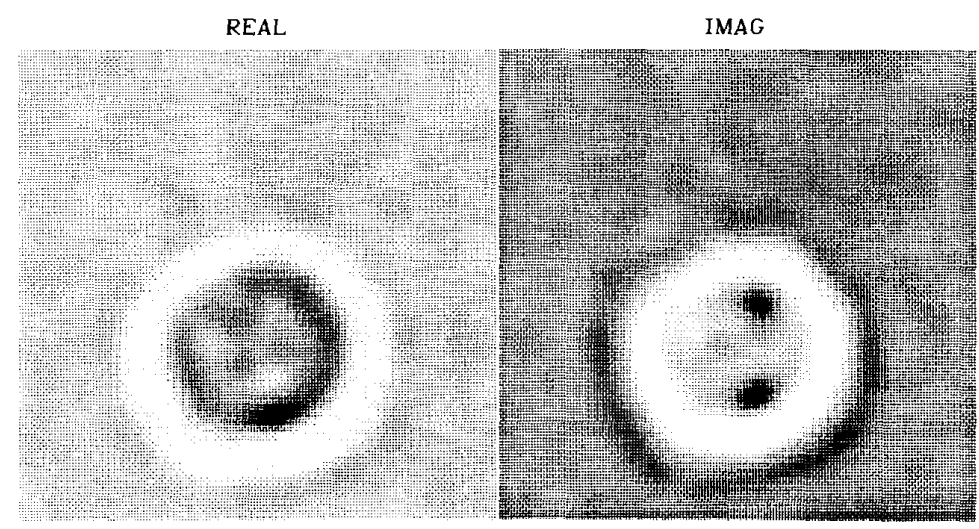

Fig. 2. Tomographic slice of a in vivo human forearm. Absolute image of complex permittivity.

tomatic network analyzer [22], [23]. Various phantoms have been constructed. Fig. 3 shows a phantom simulating a child's head. The geometry was based on X-ray CT scan data and the dielectric properties taken from the literature [24], [25]. The phantom consists of a plaster outer shell to simulate bone, with a gel simulating gray brain matter and a liquid (water and oil mixture) the properties of white brain matter. Phantoms of limbs have also been constructed.

\section{Absolute Imaging}

Absolute imaging implies taking single frame data sets to reconstruct images which contain information on the tissue structure of the object. Fig. 2 shows an absolute image of a human forearm. The pseudocolor scale represents linearly the range between minimum and maximum values in the reconstruction. The imaginary part of the complex permittivity image clearly shows the radius and ulna, the superficial fat layer and some less clearly-defined internal features. The values of permittivity are incorrectly reconstructed, due to the fact that the object falls outside the region of validity of the Born approximation. In particular the high contrast between the real part of permittivity of bone: 8 and muscle: 50 , appears as compressed between 65 and 92 around the water permittivity. For the imaginary part the effect is not so serious due to the lower contrast of the imaginary parts.

Fig. 4 shows the ideal reconstruction of the permittivity of a numerical head model. In practice the present first order algorithm does not show the internal structure of the model due to its high contrast and large size. Such problem with the algorithm effectively limit, at the moment, absolute imaging to the limbs. A large research effort is being carried out worldwide to improve reconstruction of microwave diffraction data [26]-[30], and it is hoped in the future to use this technique for absolute imaging of the brain since the substantial difference in permittivity values between different soft tissues should allow them to be easily distinguished.

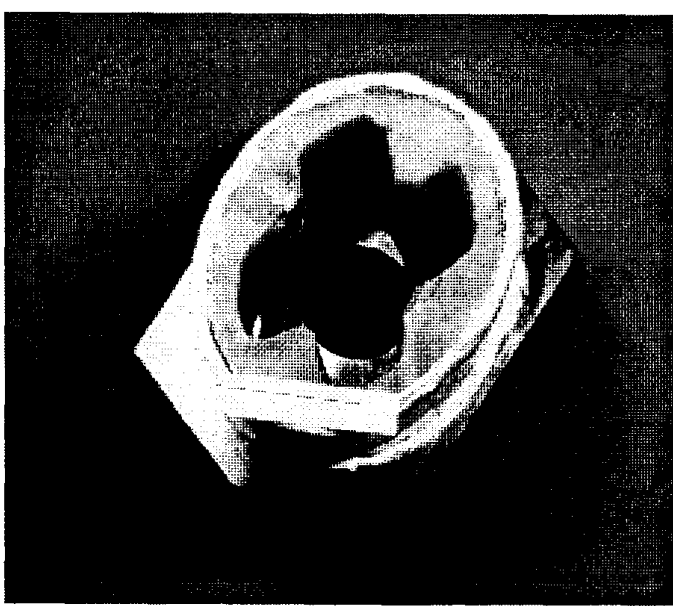

Fig. 3. Head phantom without white matter equivalent liquid.

\section{Differential Imaging}

\section{A. Thermal Change Imaging}

Fig. 5(a) show the arrangement of a preliminary experiment to estimate the temperature sensitivity of the system. A rubber tube $0.05 \mathrm{~mm}$ thick and $3 \mathrm{~cm}$ internal diameter was placed off center in the water tank. Water from an external temperature tank was pumped through the tube, whilst the surrounding medium was maintained at $T o=24^{\circ} \mathrm{C}$. Fig. $5(\mathrm{~b})$ shows a differential image corresponding to a temperature change of $\Delta T=2{ }^{\circ} \mathrm{C}$ ( $T=T o$ $+\Delta T)$, the graph, Fig. 5(c), showing the linear relationship between the differential image values and temperature change $(\Delta T)$. The temperature resolution was experimentally found to be $0.5^{\circ} \mathrm{C}$. It is expected that in a more lossy and diffracting medium such as the human body, the temperature resolution would deteriorate. However, this measured resolution is comparable with phantom results reported for other imaging techniques being considered for noninvasive temperature monitoring in hyperthermia [31], [32]. 

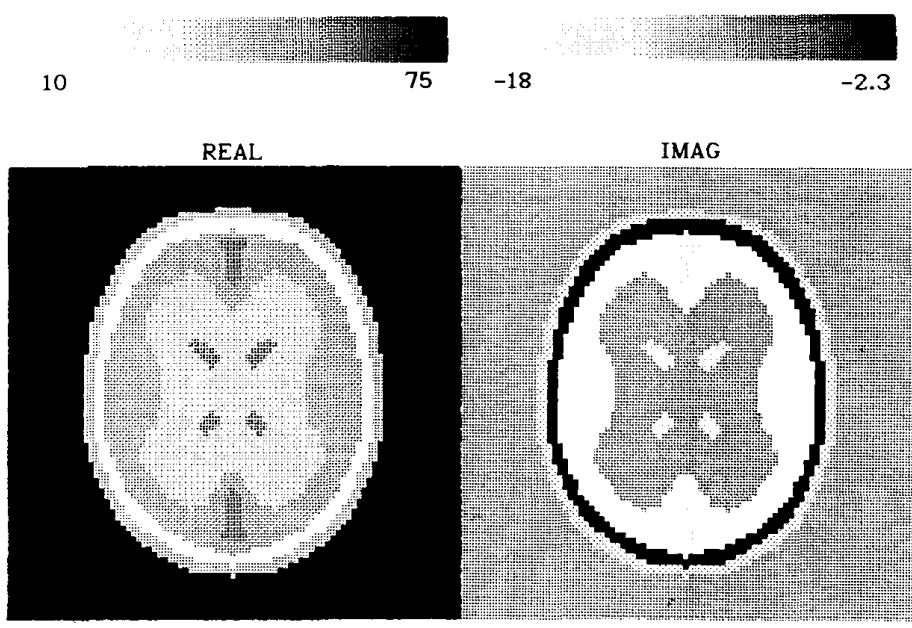

Fig. 4. Ideal image of complex permittivity corresponding to a human head numerical model [Fig. 7(a)], as would be obtained with a perfect algorithm.

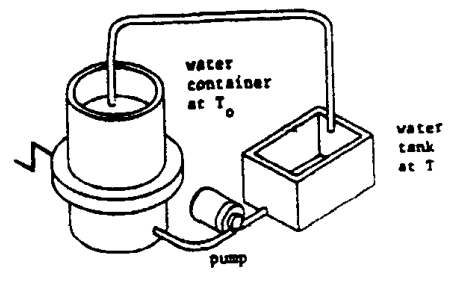

(a)

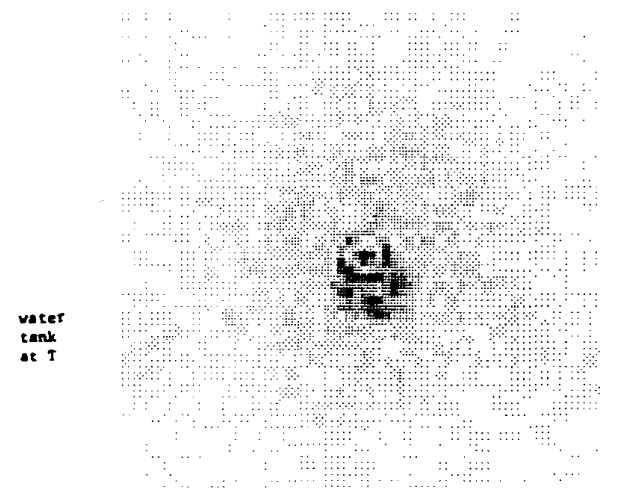

(b)

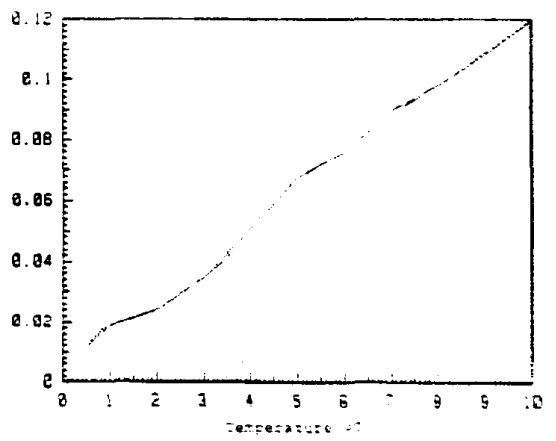

(c)

Fig. 5. (a) Experimental setup for measurement of temperature changes. (b) lmage of $2^{\circ} \mathrm{C}$ temperature change (differential image of profile magnitude). (c) Maximum value of image versus temperature of water in tube.

\section{B. Blood Content Imaging}

In order to demonstrate the capability of imaging blood content variations, the blood content of the arm of a volunteer was varied. After holding the arm in the air to remove as much blood as possible, a pressure cuff was applied to the upper arm and pressurized to above the systolic pressure. The arm was then placed in the tank and after taking three images, the cuff was released to just above the diastolic pressure thus increasing the blood content of the arm with time. Images were taken at the rate of one every three seconds for the next $90 \mathrm{~s}$. After $70 \mathrm{~s}$, the cuff pressure was released completely, allowing the blood content to begin to return to normal. The series of images (Fig. 6) shows a linear increase in the maximum image value until the cuff is released completely, when the image values began to fall. The images show a gradual development and change of position of the maxima.

\section{Differential Imaging of the Head}

Reconstructions of numerically simulated data for the head were performed. The forward scattered fields were calculated on a circular measurement array identical to that of the prototype system assuming the object is immersed in water, using an iterative conjugate gradient method [33], [34]. The numerical model of the head is identical to the phantom (Fig. 3) but includes an outer skin layer of $5 \mathrm{~mm}$ thickness. Fig. 7 shows a change in the dielectric properties of the whole brain. This differential image has been obtained by processing the change of the scattered field produced by the actual head and a reference one obtained using a priori information, in this case the contour, skin, and bone permittivities and an average permittivity for the brain. The internal form of the brain model can be recognized in the image. For Fig. 8 the permittivity values of three small areas within the brain 


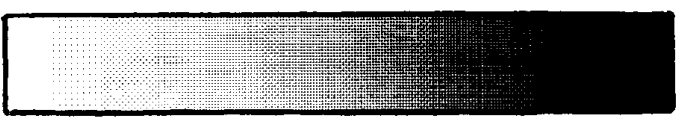

3

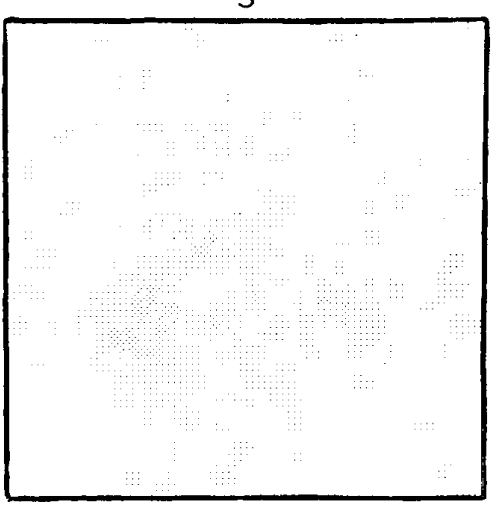

16

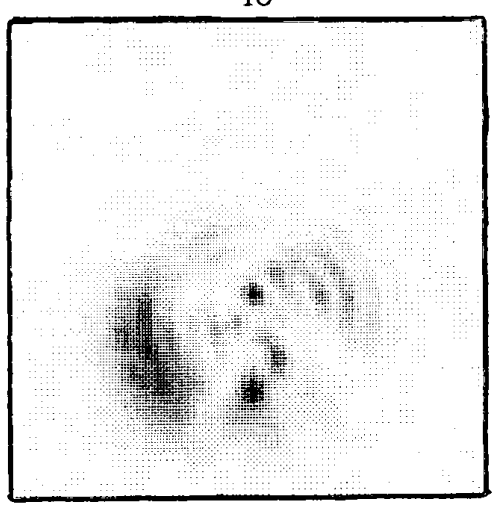

29

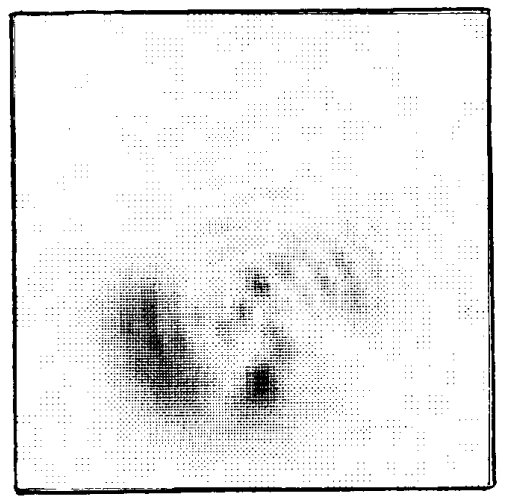

7

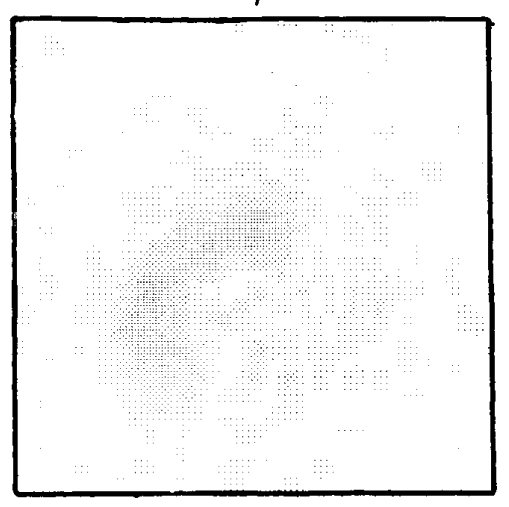

19
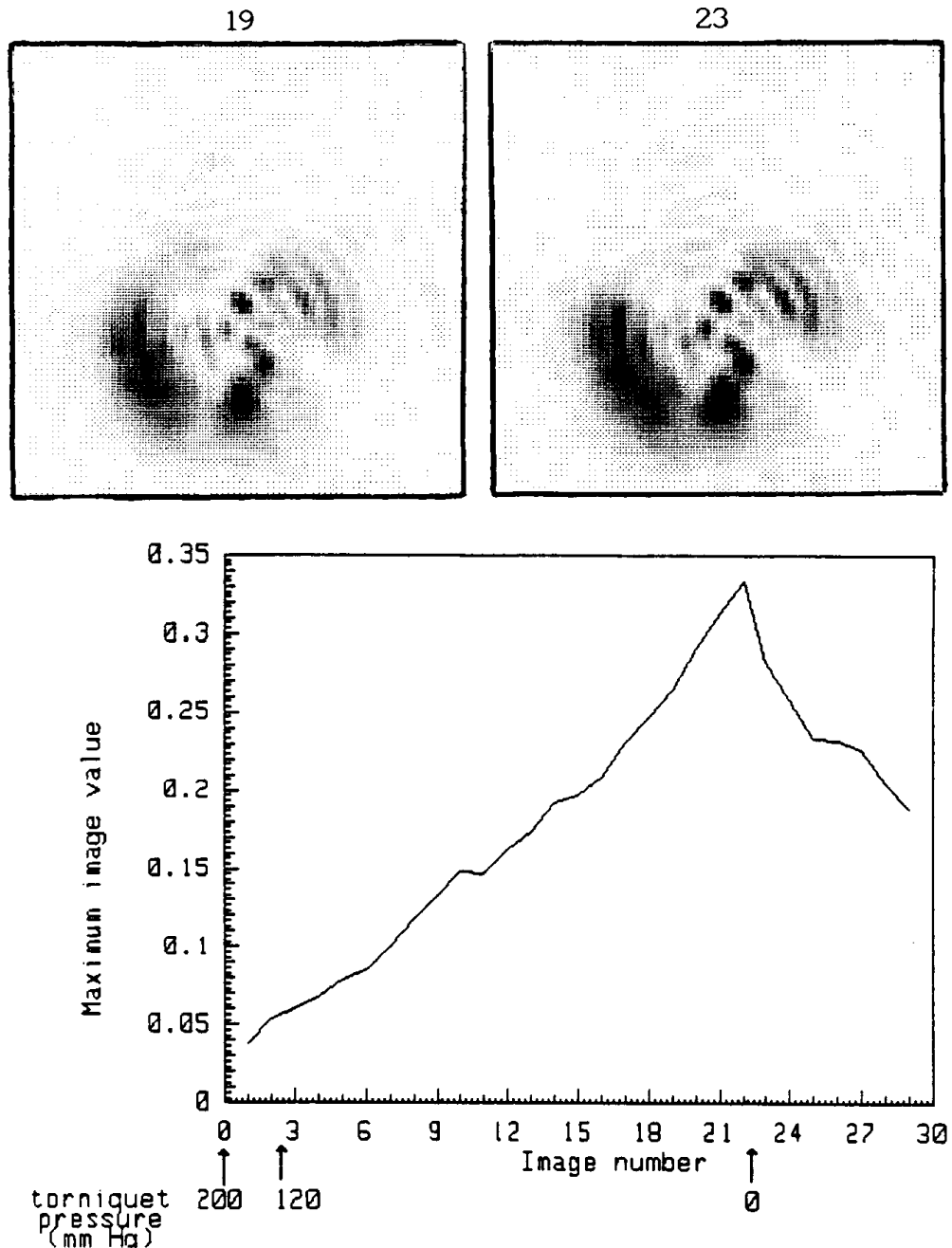

Fig. 6. A selection from a series of images of blood content of the arm (differential images of profile magnitude). The number associated with each image corresponds to it's place in the sequence from 0 to 29 , each image taking $3 \mathrm{~s}$. The basis image for these difference images is that of Fig. 2. The graph shows the maximum value of the image versus the image number and indicates the places in the sequence where the pressure in the pressure cuff was changed. 


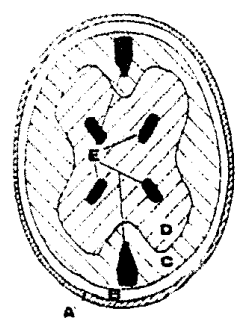

(a)

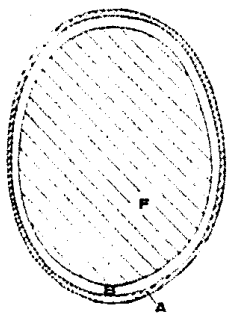

(b)

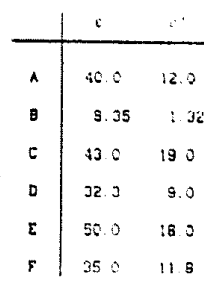

Fig. 7. Reconstructed image of the brain assuming $a$ priori knowledge (numerical simulation). (a) Model for computer simulation of the head. (b) Assumed a priori model of the head. (c) Differential image of (a)-(b) profile magnitude.

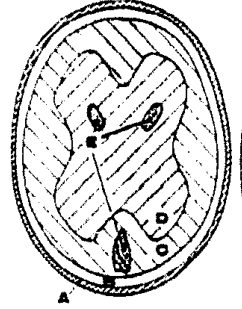

(a)

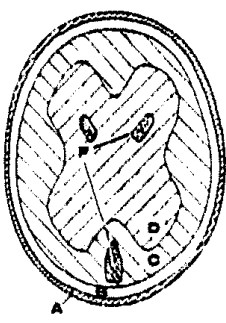

(b)

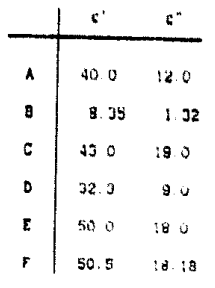

Fig. 8. Image of small ( $1 \%$ ) changes within the brain (numerical simulation). (a) Basis model (b) Model after change. (c) Reconstruction of change, differential image of profile magnitude.

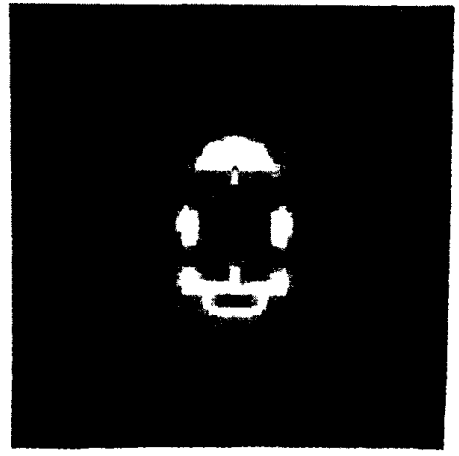

(c)

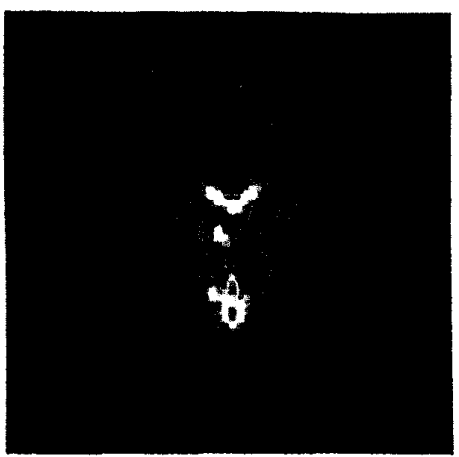

(c)

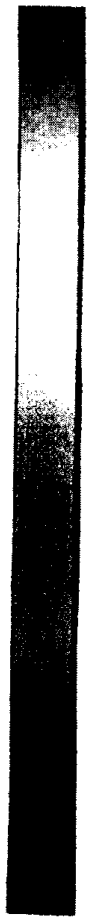

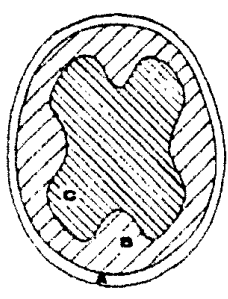

(a)

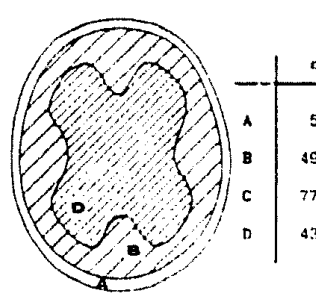

(b)

Fig. 9. Image obtained by substituting water for white matter equivalent liquid. (a) Basis phantom (b) Phantom after change. (c) Reconstruction of change, differential image of complex permittivity.

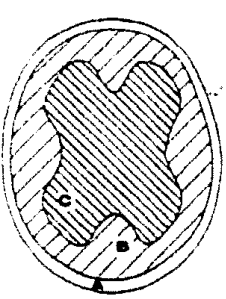

(a)

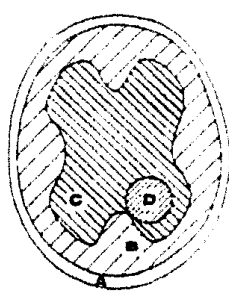

(b)

Fig. 10. Image obtained on introducing a cylindrical bar with the permittivity of blood into the white matter equivalent liquid. (a) Basis phantom. (b) Phantom with bar. (c) Reconstruction of change, differential image of profile magnitude [same scale

\begin{tabular}{l|rr} 
& \multicolumn{1}{|c}{$c^{\circ}$} & $c^{\circ}$ \\
\hline$A$ & 5.1 & 1.1 \\
8 & $\$ 9.0$ & 18.5 \\
$C$ & 33.3 & 8.0 \\
$D$ & 65.5 & 17.3
\end{tabular}

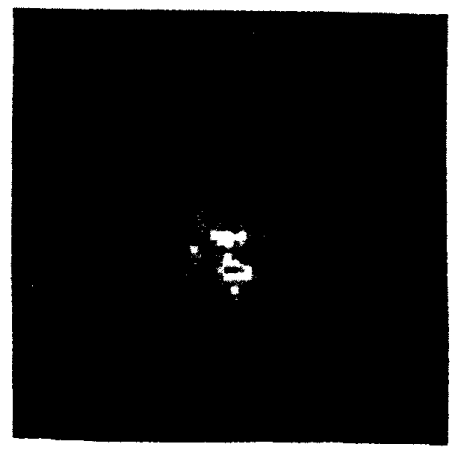

(c) 
were changed by $1 \%$, showing that small changes in the dielectric properties of the brain can, in simulations, be successfully imaged.

Fig. 9 shows a measured differential image of the head model, changing the inner section of the brain from a white matter equivalent to water. The reconstruction shows up well the extension and form of the change. In Fig. 10, a small hemorrhage in the right posterior section of the white matter is created by adding a small gel block with the dielectric properties of blood. The image represents well the position of the hemorrhage with respect to the brain visualized in Fig. 9, although with some artifacts.

\section{Discussion And Conclusion}

The results presented show the capability of the prototype system for absolute imaging of smaller biological bodies (e.g., limbs) with differential imaging of induced temperature changes and blood flow changes. Numerical simulations and measurements on a head phantom show the potentiality of the system to visualize alterations within the brain.

Possible clinical applications are being sought by the authors. Suggested applications include the visualization of blood content changes in the limbs with applications in detecting thromboses since regions with poor blood supply would show smaller changes in permittivity. A cardiac gating system is presently being added to the imaging system and should improve the imaging of blood flow. Results show that imaging changes within the brain should be possible with the present algorithms. One possible application would be to detect brain hemorrhaging in neonates, although this application is expected to bring many additional practical problems. Visualization of regional blood-flow variations within the brain due to increased specific brain activity appears feasible but will require an increase in system sensitivity. Absolute imaging of the brain will require improvement of the reconstruction algorithms.

The present system of employing a water tank is convenient for the imaging of limbs, but evidently cannot be used when the head is to be imaged. A possible solution consists of employing a bolus or water-filled bag which fits between the antennas and the head. This system was successfully employed in the early X-ray head scanners to avoid the large jump in density between the tissue and the surrounding air. As in these X-ray scanners, the bag would also serve to hold the head stationary during the scan. In a future design, the matching medium could be dispensed with altogether if contacting (possibly microstrip) antenna were placed directly in contact with the tissue.

In conclusion, a microwave scanner for rapid tomographic imaging of the body has been produced. The system has no mechanical movement and patient microwave radiation levels are below the safety standards for long exposure. The technology used is readily available and relatively inexpensive. Research will continue to improve reconstruction algorithms, increase system sensitivity, and to assess the system for clinical applications.

\section{ACKNOWLEDGMENT}

We would like to acknowledge the help of l'Ecole Supérieure d'Electricité, Paris, France, for the computer program used in the numerical simulations of the head. We also thank Dr. H. Almirall for her medical assessment in this work.

\section{REFERENCES}

[1] R. K. Mueller, M. Kaveh, and G. Wade, "Reconstructive tomography and applications to ultrasonics," Proc. IEEE, vol. 67, pp. 567587, Apr. 1979

[2] B. Duchene and W. Tabbara, "Tomographie ultrasonore par diffraction," Rev. Phys. Appl., no. 6, pp. 299-304, June 1985.

[3] D. C. Barber, B. H. Brown, and I. L. Freeston, "Imaging spatial distributions of resistivity using applied potential tomography," Electron. Lett., vol. 19, no. 22, pp. 933-934, 1983.

[4] J. Ch. Bolomey, L. Jofre, Ch. Pichot, and G. Peronnet, "Microwave diffraction tomography for biomedical applications,"' IEEE Trans. Microwave Theory Tech., vol. MTT-30, pp. 1988-2000, Nov. 1982.

[5] H. Ermert and M. Dohlus, "Microwave-diffraction-tomography of cylindrical objects using 3-dimensional wave fields," ntzArchiv, Bd. 8, H. 5, pp. 111-117, 1986.

[6] E. C. Burdette, F. L. Cain, and J. Seals, "In situ tissue permittivity at microwave frequencies: Perspective, techniques, results, " in Medical Applications of Microwave Imaging, L. E. Larsen and J. H. Jacobi, Eds. New York: IEEE, 1986, pp. 13-40.

[7] E. C. Burdette, P. G. Friedrich, R. L. Seaman, and L. E. Larsen, "In situ permittivity of canine brain: Regional variations and postmortem changes," IEEE Trans. Microwave Theory Tech., vol. MTT-34, pp. 38-50, Jan. 1986.

[8] L. Larsen and J. Jacobi, "Microwave scattering parameter imagery of an isolated canine kidney,'" Med. Phys., vol. 7, no. 1, pp. 1-7, 1980 .

[9] Ch. Pichot, L. Jofre, G. Peronnet, and J. Ch. Bolomey, "Active microwave imaging of inhomogeneous bodies," IEEE Trans. Antennas Propagat., vol. AP-33, pp. 416-425, Apr. 1985.

[10] S. J. Foti, R. P. Flam, J. F. Aubin, L. E. Larsen, and J. H. Jacobi, "A water-immersed microwave phased array system for interrogation of biological targets," in Medical Applications of Microwave Imaging, L. E. Larsen, J. H. Jacobi, Eds. New York: IEEE, 1986, pp. $148-166$.

[11] J. Ch. Bolomey, L. Jofre, and G. Peronnet, "On the possible use of microwave-active imaging for remote thermal sensing," IEEE Trans. Microwave Theory Tech., vol. MTT-31, pp. 777-781, Sept. 1983.

[12] G. Gaboriaud, C. Lavergne, J. Ch. Bolomey, M. S. Hawley, P. Berthaud, M. Gautherie, and Ch. Lavoine, "Microwave tomography in noninvasive control of hyperthermia," IEEE Eng. Med. Biol. Soc., Nov. 1987.

[13] G. Peronnet, Ch. Pichot, J. Ch. Bolomey, and L. Jofre, "A microwave diffraction tomography system for biomedical applications,", presented at 13th European Microwave Conf., Nurnberg, Sept. 1983, pp. 529-533.

[14] L. Jofre, E. de los Reyes, M. Ferrando, A. Elias, J. Romeu, M. Baquero, and J. M. Rius, "A cylindrical system for quasi-real-time microwave tomography," presented at 16th European Microwave Conf., Dublin, Sept. 1986, pp. 599-604.

[15] A. Broquetas, M. Ferrando, J. M. Rius, L. Jofre, E. de los Reyes, A. Cardama, A. Elias, and J. Ibanez, "Temperature and permittivity measurements using a cylindrical microwave imaging system," presented at 17th European Microwave Conf., Rome, Sept. 1987, pp. 892-895.

[16] A. Bioquetas, A. Elias, L. Jofre, M. Ferrando, and E. de los Reyes. "A cylindrical microwave tomographic system for medical imaging," IEEE Trans. Microwave Theory Tech., to be published.

[17] J. M. Rius, M. Ferrando, L. Jofre, and A. Broquetas, "Microwave tomography: An algorithm for cylindrical geometries," Electron. Lett., vol. 23, no. 11, pp. 564-565, 1987. 
[18] S. X. Pan and A. C. Kak, "A computational study of reconstruction algorithms for diffraction tomography: Interpolation versus filtered backpropagation," IEEE Trans. Acoust., Speech, Signal Processing, vol. ASSP-31, pp. 1962-1275, Oct. 1983

[19] A. P. Anderson and M. F. Adams, "Holographic and tomographic imaging with microwaves and ultrasound," in Inverse Methods in Electromagnetic Imaging, NATO ASI Series C, Vol. 143. WolfgangBoeruer, Eds. Dordrecht, Holland: Reidel, 1985, pp. 1077-1105.

[20] J. M. Rius, A. Broquetas. M. Ferrando, A. Cardama, and L. Jofre. "Tomografía de microondas: Reconstrucción de objetos con pérdidas en geometrías cilíndricas," in Proc. VI Meeting URSI, Valencia, Spain, Sept. 1987, pp. 511-516.

[21] G. Hartsgrove, A. Kraszewski, and A. Surowiec, "Simulated biological materials for electromagnetic absorption studies," J. Bioelec tromagn., vol. 7, 1986

[22] T. Whit Athey, M. A. Stuchly, and S. S. Stuchly, "Measurement of radio frequency permittivity of biological tissues with an open-ended coaxial line: Part I," IEEE Trans. Microwave Theory Tech., vol MTT-30, pp. 82-86, Jan. 1982.

[23] M. A. Stuchly, T. Whit Athey, G. M. Samaras, and G. E. Taylor, "Measurement of radio frequency permittivity of biological tissues with an open-ended coaxial line: part II-Experimental results, " IEEE Trans. Microwave Theory Tech., vol. MTT-30, pp. 87-92, Jan. 1982

[24] C. H. Dumey et. al. Radiofrequency Radiation Dosimetry Handbook. Univ. Utah, Rep. SAM-TR-78-22, May 1978.

[25] M. A. Stuchly and S. S. Stuchly, "Dielectric properties of biological substances-Tabulated," J. Microwave Power. vol. 15, no. 1.pp. 19-25. 1980.

[26] D. K. Ghodgaonkar, O. P. Ghandi, and M. J. Hagmann, “Estimations of complex permittivities of three-dimensional inhomogeneous bodies," IEEE Trans. Microwave Theory Tech., vol. MTT-31. pp. 442-446, June 1983.

[27] B. A. Roberts and A. C. Kak, “Reflection mode diffraction tomography," Ultrason. Imag., vol. 7, pp. 300-320, 1985.

[28] A. J. Devaney, "Reconstructive tomography with diffracting wavefields," Inverse Prob., vol. 2, pp. 161-183, 1986.

[29] L. Chommelux, Ch. Pichot, and J. Ch. Bolomey, "Electromagnetic modeling for microwave imaging of cylindrical buried inhomogeneities." IEEE Trans. Microwave Theory Tech, vol. MTT-34. Oct. 1986

[30] A. P. Anderson and R. Ait-Mchdi, "Phase tomography in microwave diagnostics," Electron. Lett., vol. 19, pp. 873-874, 1983.

[31] R. J. Dickinson, A. S. Hall, A. J. Hind, and I. R. Young, "Measurement of changes in tissue temperature using MR imaging, " $J$. Comput. Assist. Tomography. vol. 10, pp. 468-472. 1986.

[32] J. Conway, M. S. Hawley, A. D. Seagar, B. H. Brown, and D. C. Barber, "Applied potential tomography for noninvasive thermal imaging during hyperthermia treatment." Electron. Lett., vol. 21, no. 19. 1985.

[33] F. Jouvie, D. Lesselier, and D. Vuillet-Laurent, "Iterative techniques applied to some radiation and scattering problems." in Proc. Amer, Radio Sci. Meet., URSI, Vancouver, June 17-21, 1985, p. 103.

[34] P. M. Van Den Berg, "Iterative computational techniques in scattering based upon the integrated square error criterion." IEEE Trans. Antennas Propagat. vol. AP-32. pp. 1063-1071. Oct. 1984. working on antenna measurement and electromagnetic imaging. He is currently Professor and Director of the Telecommunication Engineering School at the UPC where he is engaged in research in the areas of antennas and electromagnetic imaging, both in numerical and experimental aspects.

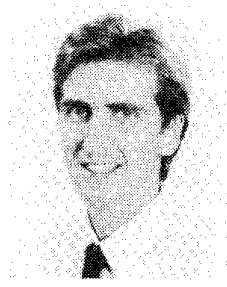

Mark S. Hawley was born in Sheffield, England, in 1961 . He received the Honors B.Sc. degree in physics in 1982 .

He joined the Department of Medical Physics, Weston Park Hospital and received the Ph.D. degree in 1986 for his work in microwave radiometry, a joint project with the Department of Electronic and Electrical Engineering. University of Sheffield

Since 1986 he has worked in the fields of noninvasive thermometry during hyperthermia treatment and microwave imaging with the Curie Institute. Supelec and SATIMO in Paris, France and with ETSIT Barcelona, Spain, where the reported work was carried out. He is currently working at the District General Hospital in Barnsley, England, developing devices to assist disabled people.

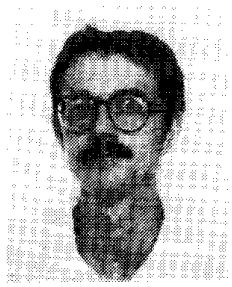

Antoni Broquetas ( $\mathrm{M}^{\prime} 90$ ) was born in Barcelona in 1959. He received the Ingeniero degree in telecommunication engineering from the Universitat Politècnica de Catalunya (U.P.C.) in 1985 and the Doctor Ingeniero degree in telecommunication engineering from the U.P.C. for his work in microwave tomography in 1989

In 1984 he joined the Electrophysics group of the U.P.C. working in microwave systems and digital radio-links. During 1986 he was a research assistant in the Portsmouth Polytechnic (U.K.) involved in the development of microwave receivers for propagation studies. Currently he is a lecturer of the Telecommunication School of Barcelona and involved in electromagnetic scattering, imaging, and radar.

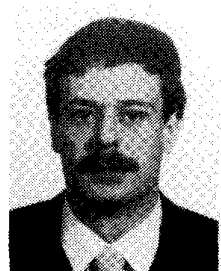

Luis Jofre ( $\mathrm{S}^{`} 79-\mathrm{M}^{`} 83$ ) was born in Mataró Spain, in 1956. He received the Ingeniero and Doctor Ingeniero degrees in telecommunication engineering, both from the Universidad Politécnica de Cataluña (UPC), in 1978 and 1982. respectively.

In 1978 he was a Research Assistant in the Electrophysics Group at UPC where he worked on analysis and near-field measurement of antennas During 1981 he joined the Ecole Supérieure d'Electricité where he was involved in microwave imaging techniques for biomedical applications. During 1986-1987 he was a Visiting Fulbrigth Scholar at Georgia Institute of Technology, Atlanta,

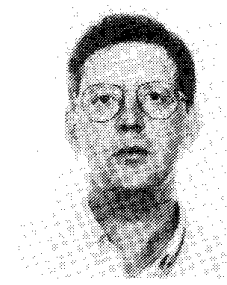

Elias de los Reyes ( $\mathrm{S}^{\prime} 78-\mathrm{M}^{\prime} 81$ ) received the Ingeniero de Telecomunicación degree from the Universidad Politécnica de Madrid (U.P.M) in 1974. In 1978 he received the Doctor Ingeniero degree from the Universidad Politécnica de Cataluña in telecommunication engineering.

In 1975 he joined the Electrophysics group of the Telecommunication School of Barcelona, becoming Full Professor in 1987. He has been involved in research in microwave systems, radar. and microwave imaging. Since October 1988 he has been in charge of the Department of Signal and Communications Theory of the Telecommunication School of Valencia (Spain). He is author and coauthor of several papers and technical reports. 


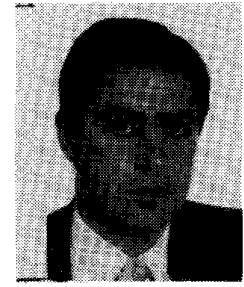

Miguel Ferrando ( $\mathrm{S}^{\prime} 78-\mathrm{M}^{\prime} 83$ ) was born in $\mathrm{Al}$ coy, Spain, in 1954. He received the Ingeniero and Doctor Ingeniero degrees in telecommunication engineering, both from the Universidad Politécnica de Cataluña (U.P.C.), in 1977 and 1982 respectively.

In 1979 he joined the "Centre National d'Etudes de Telecomunication," Lannion, France. From 1985 to the present he has been an Associate Professor at the Telecommunication Engineering School at the U.P.C. He has worked in the areas of microstrip antennas, diffraction and scattering, near-field measurements, and numerical methods.

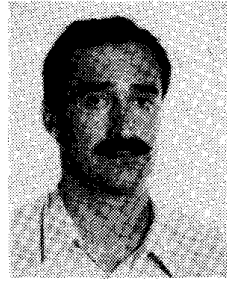

Antonio R. Elias-Fusté ( $S^{\prime} 81-M^{\prime} 82$ ) received the Ingeniero and Doctor Ingeniero degrees in telecommunication engineering, both from the Universitat Politecnica de Catalunya (U.P.C.) in 1978 and 1982 , respectively.

In 1978 he joined the Antenas-Microondas-Radar Group of the "Escuela Tecnica Superior de Ingenieros de Telecomunicacion de Barcelona" and he works in microwave characterization and RF systems design. Since 1982 he has been an Associate Professor at the U.P.C. where he is engaged in research in the field of radar. He is the author and coauthor of several reports for the industry and papers published in technical journals and conference proceedings.

Dr. Elias-Fusté is an AOC member. Currently, he is the Chairman of the AES Spanish Chapter. 